\title{
Building Genetic Database at Medical Institutes: Implement Patient Cost Audit and Improve Biomedical Research
}

\author{
Pramod Gupta $^{\mathrm{a}}$ Vipin Koushal ${ }^{\mathrm{b}}$ Chandradeo Narayan ${ }^{\mathrm{c}}$ Akshay Anand $^{\mathrm{d}}$ \\ ${ }^{a}$ Department of Biostatistics, ${ }^{b}$ Department of Hospital Administration, ${ }^{c}$ Department of Medical Microbiology, and \\ ${ }^{\mathrm{d}}$ Neuroscience Research Laboratory, Department of Neurology, Post Graduate Institute of Medical Education and \\ Research, Chandigarh, India
}

The Indian healthcare system faces multiple challenges that include unsustainable increases in cost, uneven quality of care, and persistent barriers to universal access. The costs of treatment are significant at tertiary care hospitals, and vary depending on whether the hospital is public or private, or diagnostic tests are available within or outside the hospital. Outsourcing of some or all diagnostic tests not only increases the cost of treatment for patients, but also compromises patients' data, and thus their security and confidentiality. The other serious aspect of outsourcing is the quality of diagnostics and lack of information about the exact procedures. As research and education rely on the quality of these procedures, the promise of quality treatment in medical institutes still remains an elusive goal.

The medical institute, which has all requisite facilities under one roof, is not only financially self-sustainable for patient care, but it also has the potential to provide costeffective treatment and capacity to indigenize innovative therapeutics, integrating all forms of healthcare systems into Biomedical Research. Ironically, this has not been adequately exploited even in those Indian medical Institutes which have been created with such mission and millions of rupees.

This is due to the complete absence of stated S\&T policy for medical institutes.

The clinical utility of genetic tests is now very popular at medical institutes in developed countries, particularly for untreatable disorders. Indian Medical Institutes/ Universities fail to utilize lack of genetic tests because of outsourcing, test manufacturers' inability to demonstrate clinical utility, timely availability, and misinformation about clinical establishment act. These tests are, however, known to be useful in the diagnosis and prevention of diseases. The process of genetic diagnostics is also challenging because of the rapid development in genomics research, making evidence rapidly outdated. Hence, there is an impending need for these institutes to catch up with modernization.

Indian Medical Institutes fail to translate new discoveries into practical application or generate evidence on these applications due to the outsourcing of tests. This also happens due to existing paradigms, in which, generating and evaluating evidence may be too slow, costly, and lacks data sharing. It may also be too unrepresentative to become evidence for decision makers in a timely manner. Hence, the in-house diagnostics provide a viable solution, where generating data at the cost of patient payments makes it viable and self-sustainable.

There is a dire need of policy to help build a shared genetic database so that the better and cost-effective diag-

The views expressed here are personal and do not represent those of an organisation or any office or position held by the authors.

\section{KARGER}

(C) 2017 S. Karger AG, Basel

E-Mail karger@karger.com

www.karger.com/aon
Akshay Anand

Neuroscience Research Laboratory, Department of Neurology

Post Graduate Institute of Medical Education and Research

Chandigarh 160012 (India)

E-Mail akshaylanand@rediffmail.com 
nostic testing can be made available for both patients and researchers. Such database can not only reduce the cost of Biomedical Research, but also provide better enjoinment to develop indigenous innovation and therapeutics.

It is pertinent to mention that tertiary care medical institutes of the country, providing all diagnostic facilities, could institute a policy such that a procedure for securing genetic research data from being wasted can be secured. Such government mandate will simultaneously bring down the diagnostic costs through in-house testing and will also accelerate innovation and result in indigenous solutions to healthcare delivery. Existing patient database at medical institutes are often inadequate for evaluating health interventions because of frequently missing or incorrect information, and it is thus hard to use this as an evidence for decision making.

After the audacious step of the government to demonitize the high denomination notes, there is an opportunity to mandate in-house testing regardless of the noise of "patient rush" created by some. It can be supplemented by a Quality Assurance cell to monitor the quality of treatment and research through patient cost audit. 